Check for updates

1 King's College London

2 London

Cite this as: BMJ 2021;375:n3123 http://dx.doi.org/10.1136/bmj.n3123 Published: 20 December 2021

\section{Omicron: we must protect the health and wellbeing of the public health workforce}

\author{
The Faculty of Public Health warn of fatigue and lack of recovery time as two important factors \\ affecting the public health workforce
}

Ana-Catarina Pinho-Gomes, ${ }^{1}$ Alexander Allen, ${ }^{2}$ Maggie Rae, ${ }^{2}$ Julian Ryder ${ }^{2}$

The covid-19 pandemic has had an unprecedented impact on everyone's personal and professional lives. The emergence of the omicron variant and the subsequent plan $B$ restrictions have thrown us all into a period of further uncertainty about the impact that this will have. Although not everyone was "in this together," with longstanding inequalities laid bare, the truth is that over the past two years nobody has been spared. ${ }^{1}$ Health workers, including those in public health, were arguably among the most severely affected. ${ }^{23}$ Therefore, the Faculty of Public Health (FPH), together with trainees, surveyed its members in April and May 2021 to understand the impact of the pandemic on the mental and physical health and wellbeing of its members. ${ }^{4}$

We found that a majority of our members reported feeling fatigued, with about half saying they felt emotionally exhausted, overwhelmed, or frustrated, often or always. Poor mental health and wellbeing had a more marked impact on respondents' personal rather than professional lives, negatively affecting hobbies and leisure activities, family time, sleep, and life satisfaction in general. The main causes of poor mental health and wellbeing were work stress and an unmanageable workload, the government's response to the pandemic, social isolation, uncertainty about the future, and limited ability to influence decision making in the pandemic response. Most participants ranked their physical health as very good or good, and physical health had, in general, a lesser impact on personal and professional activities than mental health. Diet, physical activity, and alcohol intake all deteriorated to a degree, but participants found a wide range of coping mechanisms for support.

Trainees reported mixed experiences regarding the impact of the pandemic on their training. The most common consequences that the pandemic has had on trainees is a reprioritisation of projects to focus on covid-19, fewer networking opportunities, increased opportunities to improve knowledge and skills in health protection, fewer opportunities to attend courses, and less variety of projects. Redeployment and extension of training time were rare in public health training, in contrast to what happened in acute medical specialties. For consultants, the pandemic brought a substantial increase in workload and diversion to the acute response to covid-19. About half experienced uncertainty owing to ever changing guidance from central authorities, changes to their formally agreed job plan, and taking on unfamiliar roles. On a positive note, others noted they had further opportunities to improve their health protection skills and developed new collaborative networks with colleagues-key skills for tackling future public health emergencies.

We heard that a lot of our members found it challenging to cope with the pandemic; most of these used walking and hiking, watching TV, chatting with friends and relatives as coping mechanisms. Although only $16 \%$ of all participants accessed formal mental health and wellbeing support, which most found useful, it is concerning that $10 \%$ of consultants and $30 \%$ of trainees did not know what support was available, and suggests that these forms of support need to be more readily available, especially as we face further uncertainty with the omicron variant. As regards the unprecedented and sustained increase in workload, more than half of participants, unsurprisingly, asked for extended annual leave and flexible working. Alongside the large scale return of previously retired members of the workforce, employers will need to be flexible and adaptable in managing working patterns for their staff in the future.

Overall, what we found compellingly illustrated that the covid-19 pandemic had a big impact on the mental and physical health and wellbeing of the public health workforce, which then had wider consequences for their personal and professional lives. There were two broad cross-cutting themes-fatigue and the need to recover. However, we saw less evidence of burnout among trainees as reported by other specialties. ${ }^{5}$ The pandemic also brought into sharp focus the importance of a healthy work-life balance, which may underpin the desire for flexible working patterns, while also contributing to sustainable lifestyles. ${ }^{6}$ In addition, covid-19 had a big, yet variable, impact on the careers of public health trainees and consultants, with trainees more optimistic than consultants. The dramatic changes to the public health infrastructure amid an ongoing pandemic, together with a heavily centralised pandemic response that left many feeling powerless, undervalued, and unable to influence decision making, were identified as drivers of poor mental health and wellbeing. Nonetheless, trainees as well as consultants agreed that the pandemic had a silver lining by offering unexpected opportunities to improve health protection skills and foster new partnerships with stakeholders and communities.

The public health workforce, have unflinchingly and continuously supported the covid-19 response in the UK for many months. However, as we now prepare to deal with the impact of the omicron variant, we 
must also allow public health professionals time to recover and enable them to tackle the challenges ahead in the next few months and the pandemic aftermath, from tackling longstanding health inequalities to fending off emerging threats, environmental degradation, and climate change.

Competing interests: none declared

Provenance and peer review: not commissioned, not peer reviewed

1 The Health Foundation. The same pandemic, unequal impacts. https://www.health.org.uk/newsand-comment/charts-and-infographics/same-pandemic-unequal-impacts

2 O'Dowd A. NHS staff's stress levels rose last year as covid pandemic took its toll. BMJ 2021;372:n703. doi: 10.1136/bmj.n703. pmid: 33712446

3 Mehta S, Machado F, Kwizera A, etal. COVID-19: a heavy toll on health-care workers. Lancet Respir Med 2021;9:226-8. doi: 10.1016/S2213-2600(21)00068-0. pmid: 33556317

4 The Faculty of Public Health. https://www.fph.org.uk/

5 Rimmer A. Covid-19: One third of trainees are affected by burnout, GMC survey finds. BMJ 2021;374:n1884. doi: 10.1136/bmj.n1884. pmid: 34315729

6 Marteau TM, Chater N, Garnett EE. Changing behaviour for net zero 2050. BM/2021;375:n2293. doi: 10.1136/bmi.n2293. pmid: 34615652 